\title{
Chiral torsional effect with finite temperature, density, and curvature
}

\author{
Shota Imaki॰ and Zebin Qiu॰ \\ Department of Physics, The University of Tokyo, Tokyo 113-0033, Japan
}

(Received 23 April 2020; accepted 8 June 2020; published 6 July 2020)

\begin{abstract}
We scrutinize the novel chiral transport phenomenon driven by spacetime torsion, namely, the chiral torsional effect (CTE). We calculate the torsion-induced chiral currents with finite temperature, density, and curvature in the most general torsional gravity theory. The conclusion complements the previous study on the CTE by including curvature and substantiates the relation between the CTE and the Nieh-Yan anomaly. We also analyze the response of chiral torsional current to an external electromagnetic field. The resulting topological current is analogous to that in the axion electrodynamics.
\end{abstract}

DOI: 10.1103/PhysRevD.102.016001

\section{INTRODUCTION}

A prominent feature of relativistic chiral matter is the existence of various novel chiral transport phenomena. Famous examples are the chiral magnetic effect (CME) and the chiral vortical effect (CVE), i.e., the generation of electric current along a magnetic field and vorticity, respectively, on the condition of chirality imbalance [1-4]. The chirality imbalance is produced by virtue of the axial anomaly [5] so that these macroscopic chiral transport phenomena are associated with the underlying quantum anomaly. It has been demonstrated that the CME is related to the axial anomaly [6]. Meanwhile, the CVE has been regarded as also involving the gravitational anomaly [7-9]. As observable manifestations of the quantum anomaly and topological properties of chiral gauge theories, chiral transport phenomena have been studied with immense efforts in various physical contexts, e.g., quark-gluon plasma in heavy-ion collisions [10-15]; topological condensed matter systems such as topological insulators [16-21] or Dirac and Weyl semimetals [22-27]; electroweak media in neutron stars [28-32], the primordial universe [33-35], or core-collapse supernovae [36-38].

Very recently, a rather new type of chiral transport phenomenon was discovered. It is induced by the spacetime torsion in the presence of chirality imbalance and naturally termed the "chiral torsional effect" (CTE) [39]. Torsion is a hypothetical spacetime property in the augmented gravity theory called Einstein-Cartan gravity, which has received much attention in gravity physics as reviewed by Refs. [40,41]. More research interest stems from the idea

Published by the American Physical Society under the terms of the Creative Commons Attribution 4.0 International license. Further distribution of this work must maintain attribution to the author(s) and the published article's title, journal citation, and DOI. Funded by SCOAP. that the CTE is supposed to be connected to the Nieh-Yan anomaly [42] which depicts the torsional topology of spacetime $[43,44]$.

Although never observed in real spacetime so far, torsion can be imitated by a lattice dislocation. This idea is formulated in lattice field theory and buttressed by numerical computation in Ref. [45]. In condensed matter, torsion is realizable in diverse materials like graphene [46], topological insulators [47-49], and Weyl semimetals [50-54], where the deformation of the materials effectively acts as torsion. In particular, Weyl semimetals are ideal candidates for the CTE experiments since they bear a chirality imbalance as well.

Despite its profound theoretical significance and promising experimental verifiability, to the best of our knowledge, the previous studies of the CTE are incomplete in the sense that they have neglected curvature effectsspecifically, the spin connection term in the covariant derivative - and overlooked a certain torsional term allowed in the general torsional gravity Lagrangian. In addition, the connection between the CTE and the NiehYan anomaly is not entirely clear. Firm computation in a complete setup is indispensable for comprehending the interplay between torsion, curvature, and axial anomaly. Hence in this paper, we decisively calculate the CTE current at finite temperature, density, and curvature in the most general torsional gravity theory. In addition, we analyze the current driven by the electromagnetic field in torsional spacetime, unveiling the impact of torsion on the conventional Maxwell electrodynamics.

This paper is organized as follows. Section II serves as a brief review of torsional gravity. We introduce the basic notion of torsion and expound the general form of the coupling between torsion and a fermion. In Sec. III, we calculate the torsion-induced current. We first evaluate the current at zero temperature and density to clarify its relation to the Nieh-Yan's torsional topological invariant and then 
generalize our calculation to finite temperature and density. In Sec. IV, we analyze the current driven by electromagnetic fields in the presence of torsion and hereby illuminate the analogy between the electrodynamics of the torsional gravity theory to the axion electrodynamics. Section V presents our summary and outlook. Throughout this work, we adopt an imaginary time coordinate or, in other words, a metric with a Euclidean signature.

\section{TORSION}

The standard Einstein gravity theory assumes the symmetry of affine connection $\Gamma_{\mu \nu}^{\lambda}=\Gamma_{\nu \mu}^{\lambda}$. Together with the metricity condition, this assumption leads one to identify the affine connection with the Christoffel symbol determined solely by the metric

$$
\Gamma_{\mu \nu}^{\lambda}=\frac{1}{2} \Delta_{\rho \mu \nu}^{\alpha \beta \gamma} g^{\rho \lambda} \partial_{\alpha} g_{\beta \gamma},
$$

with a permutation symbol $\Delta_{\mu \nu \rho}^{\alpha \beta \gamma} \equiv \delta_{\rho}^{\alpha} \delta_{\mu}^{\beta} \delta_{\nu}^{\gamma}+\delta_{\nu}^{\alpha} \delta_{\mu}^{\beta} \delta_{\rho}^{\gamma}-$ $\delta_{\mu}^{\alpha} \delta_{\nu}^{\beta} \delta_{\rho}^{\gamma}$. By contrast, the Einstein-Cartan gravity theory relaxes the assumption of a symmetric affine connection, allowing for an antisymmetric part termed "torsion":

$$
T_{\mu \nu}^{\lambda} \equiv \tilde{\Gamma}_{\mu \nu}^{\lambda}-\tilde{\Gamma}_{\nu \mu}^{\lambda}
$$

Henceforth, we attach a tilde in denoting quantities containing torsion. Affine connection itself is not a tensor, but torsion is; thus, it is qualified as a physical quantity. Using the metricity condition, the relation between $\tilde{\Gamma}_{\mu \nu}^{\lambda}$ and $\Gamma_{\mu \nu}^{\lambda}$ reads

$$
\tilde{\Gamma}_{\mu \nu}^{\lambda}=\Gamma_{\mu \nu}^{\lambda}-\frac{1}{2} \Delta_{\rho \mu \nu}^{\alpha \beta \gamma} g^{\lambda \rho} T_{\alpha \beta \gamma} .
$$

Equation (3) demonstrates that the spacetime features two independent intrinsic properties: metric and torsion.

Correspondingly, the covariant derivative of a spinor field comprises an extra term embodying the coupling of torsion with a fermion:

$$
\tilde{\nabla}_{\mu} \psi \equiv \nabla_{\mu} \psi+\frac{i}{16} \Delta_{\nu \rho \mu}^{\alpha \beta \gamma} T_{\alpha \beta \gamma}\left(e_{a}^{\nu} e_{b}^{\rho}-e_{b}^{\nu} e_{a}^{\rho}\right) \sigma^{a b} \psi,
$$

where $e_{m}^{\mu}$ is the vierbein satisfying the orthonormal relations $e_{m}^{\mu} e_{\mu n}=\delta_{m n}, e_{\mu m} e_{\nu}^{m}=g_{\mu \nu}$. The first term in Eq. (4) is the torsion-free covariant derivative in Einstein gravity theory:

$$
\nabla_{\mu} \psi \equiv \partial_{\mu} \psi+\frac{i}{2} \omega_{\mu a b} \sigma^{a b} \psi,
$$

with $\sigma^{a b} \equiv \frac{i}{2}\left[\gamma^{a}, \gamma^{b}\right]$ and the spin connection $\omega_{\mu a b}=\frac{1}{4}\left(e_{b \sigma} \partial_{\mu} e_{a}^{\sigma}-e_{a \sigma} \partial_{\mu} e_{b}^{\sigma}\right)+\frac{1}{4} \Gamma_{\beta \mu}^{\alpha}\left(e_{a}^{\beta} e_{b \alpha}-e_{b}^{\beta} e_{a \alpha}\right)$.

With the covariant derivative defined by Eq. (4), we readily write down the Dirac Lagrangian in torsional curved spacetime,

$$
\mathcal{L}_{\min }=\frac{1}{2} \bar{\psi}\left(\gamma^{\mu} \tilde{\nabla}_{\mu}-m\right) \psi+\text { H.c. },
$$

which is sometimes called the minimal theory. After some algebra [41], we rewrite Eq. (7) to sort out the torsional contribution,

$$
\mathcal{L}_{\min }=\bar{\psi}\left[\gamma^{\mu}\left(\nabla_{\mu}-\frac{1}{8} \gamma_{5} S_{\mu}\right)-m\right] \psi
$$

where $S^{\mu}$ is what we call "screw torsion":

$$
S_{\mu} \equiv \varepsilon^{\mu \nu \rho \sigma} T_{\nu \rho \sigma}
$$

with $\varepsilon^{\mu \nu \rho \sigma}$ denoting the covariant Levi-Civita tensor. The most general Lagrangian obeying covariance, locality, renormalizability, and parity symmetry allows for another type of torsional term that we call "edge torsion,"

$$
E_{\mu} \equiv T_{\mu \alpha}^{\alpha}
$$

and it takes the form

$$
\mathcal{L}=\bar{\psi}\left[\gamma^{\mu}\left(\nabla_{\mu}-\eta_{1} \gamma_{5} S_{\mu}-\eta_{2} E_{\mu}\right)-m\right] \psi .
$$

The parameters $\eta_{1}$ and $\eta_{2}$ are arbitrary real numbers for the general theory, while the specific choice $\eta_{1}=1 / 8, \eta_{2}=0$ recovers the minimal theory (8).

Two features of the Lagrangian (11) play essential roles in later computation. First, the torsional terms are entirely separated. Thus, we can conveniently define the perturbation away from the torsion-free theory that corresponds to the choice $\eta_{1}=\eta_{2}=0$. Second, the edge torsion couples to a fermion in the same way as a U(1) gauge field. It enables us to easily encompass an external electromagnetic field by combining it with the edge torsion:

$$
A_{\mu}^{\prime} \equiv A_{\mu}+\eta_{2} E_{\mu}
$$

In this way, we consider $E_{\mu}$ together with the electromagnetic field in Sec. IV. Until then we turn off $A_{\mu}^{\prime}$ for simplicity.

\section{TORSION-INDUCED CURRENT}

We aim to evaluate the torsion-induced chiral current in the most general theory (11) with metric and torsion treated as background fields. Our calculation starts from the 
following vacuum or thermal expectation value of the chiral current:

$$
J_{ \pm}^{\mu}=\left\langle\bar{\psi} \gamma^{\mu} P_{ \pm} \psi\right\rangle
$$

where "+" and "-" stand for right-handedness and lefthandedness, respectively, and $P_{ \pm} \equiv \frac{1}{2}\left(1 \pm \gamma_{5}\right)$ denotes the chiral projector.

Throughout the present section, as explained above, the electromagnetic field, together with the edge torsion, $A_{\mu}^{\prime}$, is shut down, and the screw torsion $S_{\mu}$ is disposed as a perturbation to the linear order. In parallel, the effect of curvature is also kept to the leading order in terms of the curvature tensor $R_{\mu \nu \rho \sigma}$.

The chiral current $J_{ \pm}^{\mu}$ is calculated in two different setups. The result at zero temperature and density is achieved in Sec. III A. The axial current, in this case, depends on the ultraviolet cutoff, and its divergence proves to be related to the Nieh-Yan topological invariant. Then, the generalization to finite temperature and density is accomplished in Sec. III B. The chiral current relies on the interplay between torsion and curvature and exhibits a distinctive dependence on temperature and density in contrast to the CME and the CVE.

\section{A. Zero temperature and density}

At zero temperature and density, given that the screw torsion $S_{\mu}$ is an axial vector, the vector current vanishes at $\mathcal{O}\left(S_{\mu}\right)$. We therefore focus on the axial current

$$
J_{5}^{\mu}=\left\langle\bar{\psi} \gamma^{\mu} \gamma_{5} \psi\right\rangle .
$$

We calculate it as the trace involving the propagator, in a way similar to Ref. [45]. The perturbative expansion with respect to the screw torsion gives rise to

$$
J_{5}^{\mu}=-\eta_{1} \operatorname{Tr}\left(\gamma^{\mu} \gamma_{5} G \gamma^{\nu} \gamma_{5} G\right) S_{\nu}+\mathcal{O}\left(S_{\mu}^{2}, \partial^{2} S_{\mu}\right),
$$

with $G$ representing the torsion-free propagator and $\operatorname{Tr}$ standing for the trace over both Dirac indices and coordinate space. We make two remarks about our power counting. First, given the symmetry property of the curvature tensor, the torsion-independent part vanishes at zero temperature and density, as also pointed out in Ref. [4]. Second, from the perspective of parity, one can understand that the first-order derivative of $S^{\mu}$ does not contribute to the axial current.

To simplify our computation, we employ the Riemann normal coordinate around the point $x$ at which the current is evaluated. In this coordinate system, the Christoffel symbol $\Gamma_{\nu \rho}^{\mu}$ vanishes at $x$, and the $\gamma$-matrices are those in flat spacetime. After the transformation into momentum $k$-space, the propagator at the coincidental point acquires the following form according to Ref. [55]:

$$
G\left(x, x^{\prime} \rightarrow x\right)=\int \frac{d^{4} k}{(2 \pi)^{4}}\left(i \gamma^{\mu} k_{\mu}+m\right) \mathcal{G}(k),
$$

where the function $\mathcal{G}(k)$ includes curvature effects in a perturbative way:

$$
\begin{aligned}
\mathcal{G}(k)= & -\left[1-\left(A_{1}+i A_{1 \alpha} \frac{\partial}{\partial k_{\alpha}}-A_{1 \alpha \beta} \frac{\partial^{2}}{\partial k_{\alpha} \partial k_{\beta}}\right) \frac{\partial}{\partial m^{2}}\right. \\
& \left.+A_{2}\left(\frac{\partial}{\partial m^{2}}\right)^{2}\right] \frac{1}{k^{2}+m^{2}}+\cdots .
\end{aligned}
$$

The first coefficient $A_{1}$ is proportional to the scalar curvature,

$$
A_{1}=\frac{R}{12}
$$

The subsequent coefficients, $A_{1 \alpha}, A_{1 \alpha \beta}, A_{2}$ and so forth, consist of higher orders of curvature or derivatives thereof. One can refer to Ref. [55] for their specific values, but we focus on the leading-order curvature effect so that $A_{1}$ suffices.

Inserting Eqs. (16) and (17) into Eq. (15) and taking the trace over Dirac indices yields

$$
J_{5}^{\mu}=2 \eta_{1} S^{\mu} \int^{\Lambda} \frac{d^{4} k}{(2 \pi)^{4}}\left(2 m^{2}-k^{2}\right) \mathcal{G}^{2}(k) .
$$

We have introduced the ultraviolet cutoff $\Lambda$ so as to figure out the dependence of the axial current on $\Lambda$, which is also implied in Refs. $[43,44]$. With detailed computation given in Appendix A, we present the conclusive result

$$
\begin{aligned}
J_{5}^{\mu}= & \frac{\eta_{1}}{8 \pi^{2}} S^{\mu} \\
& \times\left[-\Lambda^{2}-3 m^{2}+\frac{5}{12} R+\left(4 m^{2}-\frac{R}{6}\right) \log \left(1+\frac{\Lambda^{2}}{m^{2}}\right)\right] .
\end{aligned}
$$

Here, the cutoff $\Lambda$ has definite physical significance as the inverse of lattice spacing which is the physical length scale of a crystal.

Let us examine the axial anomaly indicated by Eq. (20). To this end, we take the massless limit $m \rightarrow 0$. Furthermore, since the curvature is independent of torsion and irrelevant to our study, we rightfully take $R_{\mu \nu \rho \sigma}=0$. Then, the axial current reads

$$
J_{5}^{\mu}=-\frac{\eta_{1} \Lambda^{2}}{8 \pi^{2}} S^{\mu}
$$

Accordingly, the divergence of the axial current takes the form of [42]

$$
\partial_{\mu} J_{5}^{\mu}=\frac{\eta_{1} \Lambda^{2}}{8 \pi^{2}} \varepsilon^{\mu \nu \rho \sigma} T_{\mu \nu}^{\alpha} T_{\alpha \rho \sigma}
$$


In fact, the volume integral of the divergence is proportional to Nieh-Yan's topological invariant [42],

$$
N_{\mathrm{NY}}=\int d^{4} x \varepsilon^{\mu \nu \rho \sigma} T_{\mu \nu}^{\alpha} T_{\alpha \rho \sigma},
$$

which characterizes the torsional topology of spacetime. The relation (22) is referred to as the Nieh-Yan anomaly $[43,44]$ in that the right-hand side has an anomalous nature and the left-hand side embodies Nieh-Yan's topological invariant.

It is noteworthy that in a general sense, the divergence of the axial current in a torsional curved spacetime receives other contributions in addition to Eq. (22), which we are nevertheless unable to capture under our truncation scheme. For instance, Nieh-Yan's topological invariant should have the Pontryagin form of the curvature [42], together with Eq. (23), which is at the second order of the curvature tensor. As first indicated in Ref. [56], there is also a $\Lambda$-independent torsional contribution to the axial anomaly from higher orders of torsion and its derivative. To grasp this, one can extend our analysis to include higher-order terms of curvature and torsion.

\section{B. Finite temperature and density}

We now generalize to the chiral current (13) at finite temperature $T$, vector chemical potential $\mu$, and axial chemical potential $\mu_{5}$. For such purposes, we resort to the Matsubara formalism. We impose the stationary condition of the metric; i.e., all metric components are time independent, and the temporal components are space independent, which justifies the standard Matsubara formalism. For simplicity, we consider a massless fermion with $m=0$.

We observe from the Lagrangian (11) that the temporal component of the screw torsion couples to a fermion in a way identical to the axial chemical potential. Thus, we absorb it into a redefined axial chemical potential:

$$
\mu_{5}^{\prime} \equiv \mu_{5}+\eta_{1} S_{\tau}
$$

Then, without loss of generality, we specify the screw torsion to be pure spacelike and further direct it along the $z$ axis as $S_{\mu}=S_{z} \hat{z}$ because of spherical symmetry. One can manifest that only the $\tau$ - and $z$-components of the current (13) are nonvanishing. Since the $\tau$-component does not depend on $S_{z}$ at the linear order, we focus on the z-component,

$$
J_{ \pm}^{z}=\left\langle\bar{\psi} \gamma^{z} P_{ \pm} \psi\right\rangle
$$

It is straightforward to prove that the current (25) can be evaluated by a formula similar to Eq. (15), with the momentum $k_{\mu}$ therein replaced by

$$
K_{ \pm \mu} \equiv\left(\boldsymbol{k}, \omega_{n}+i \mu_{ \pm}\right)
$$

with the Matsubara frequencies $\omega_{n} \equiv 2 \pi T\left(n+\frac{1}{2}\right)$ and the chiral chemical potential $\mu_{ \pm} \equiv \mu \pm \mu_{5}^{\prime}$. To linear order, the chiral current is expressed as

$$
J_{ \pm}^{z}=-\eta_{1} \operatorname{Tr}\left(\gamma^{z} P_{ \pm} G_{ \pm} \gamma^{z} \gamma_{5} G_{ \pm}\right) S^{z}+\cdots,
$$

where $G_{ \pm}$is given by

$$
G_{ \pm}=T \sum_{\omega_{n}} \int \frac{d^{3} \boldsymbol{k}}{(2 \pi)^{3}} i \gamma^{\mu} K_{ \pm \mu} \mathcal{G}_{ \pm}(k)
$$

with the perturbative expansion of $\mathcal{G}_{ \pm}(k)$ formally similar to Eq. (17),

$$
\mathcal{G}_{ \pm}(k)=-\frac{1}{K_{ \pm}^{2}}-\frac{R}{12} \frac{1}{\left(K_{ \pm}^{2}\right)^{2}}+\cdots
$$

Applying the formulas (28) and (29) to the expression (27) and carrying out the Dirac trace, the computation can be condensed to the following sum integral:

$$
J_{ \pm}^{z}= \pm 2 \eta_{1} S^{z} T \sum_{\omega_{n}} \int \frac{d^{3} \boldsymbol{k}}{(2 \pi)^{3}}\left(2 k_{z}^{2}-K_{ \pm}^{2}\right) \mathcal{G}_{ \pm}^{2}(k)
$$

Now that we are interested in the dependence of $J_{ \pm}^{z}$ on temperature and density rather than the ultraviolet scale, we calculate the integral with dimensional regularization and subtract the divergence according to the modified minimal subtraction scheme. After the computation of the sum integral detailed in Appendix B, we obtain the final result:

$$
J_{ \pm}^{z}= \pm \frac{\eta_{1} R}{96 \pi^{2}} S^{z} F\left(\frac{\mu_{ \pm}}{2 \pi T}\right) .
$$

The dependence on temperature and density is expressed utilizing the digamma function $\psi(z)$ as

$$
F(z)=\psi\left(\frac{1}{2}+i z\right)+\psi\left(\frac{1}{2}-i z\right),
$$

which is depicted in Fig. 1. One can further compute the vector and axial currents from Eq. (31). Notably, the vector current vanishes when there is no chiral imbalance, $\mu_{5}=0$.

We remark that the result (31) should not be directly compared with that in zero temperature and density (20) because the result of $J_{ \pm}^{\mu}$ would change by altering the order in which we take the three limits, $T \rightarrow 0, \mu_{ \pm} \rightarrow 0$, and $m \rightarrow 0$. For example, in Eq. (31), the $\mu_{ \pm} \rightarrow 0$ limit can be taken directly, while the $T \rightarrow 0$ limit should be analyzed through the asymptotic expansion; apparently, the results have different coefficients of $R S^{z}$. A discussion on the $T \rightarrow 0$ limit is provided in Appendix B. Moreover, during 


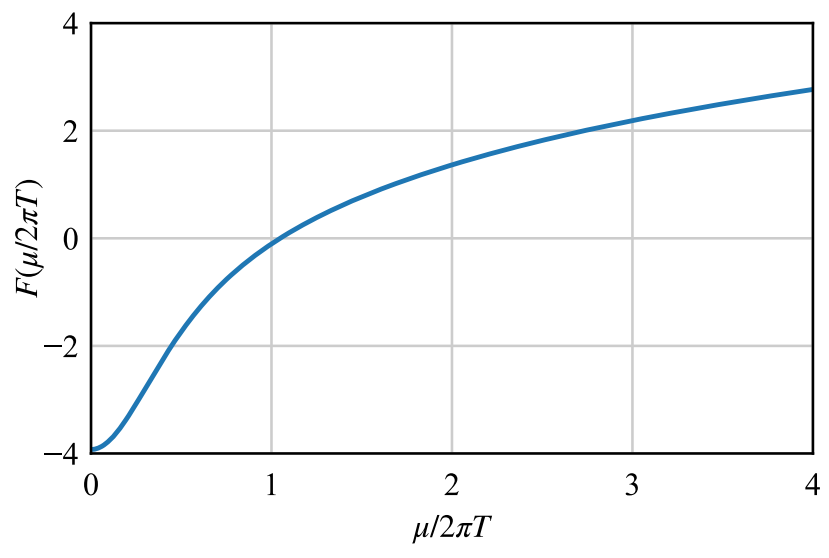

FIG. 1. The dependence of the function $F$ in Eq. (31) on temperature and density.

dimensional regularization, an infinite portion in $J_{ \pm}^{z}$ exists as the counterpart of the $\Lambda$-dependent term in Eq. (20), but it has already been subtracted and is thus absent in Eq. (31).

\section{TORSIONAL ELECTRODYNAMICS}

Now, we study the current response to an external electromagnetic field in the presence of torsion. It is worth noting that we combine the edge torsion with the electromagnetic field as

$$
A_{\mu}^{\prime} \equiv A_{\mu}+\eta_{2} E_{\mu}
$$

Hence, our analysis in this section accounts for the current driven by the edge torsion as well. For simplicity, we confine our study to the massless fermion on a flat metric with zero chemical potential. We also assume the screw torsion to be stationary and homogeneous. Under these assumptions, we can perform an axial transformation

$$
\psi(x) \rightarrow \exp \left(-i \eta_{1} \gamma_{5} S_{\mu} x^{\mu}\right) \psi(x)
$$

to eliminate $S_{\mu}$ from the fermionic sector of the Lagrangian. This transformation meanwhile yields the following anomalous term in the gauge sector:

$$
S_{\text {anom }}=\frac{\eta_{1}}{4 \pi^{2}} \int d^{4} x A_{\mu}^{\prime} S_{\nu} \tilde{F}^{\mu \nu}
$$

where $F_{\mu \nu} \equiv \partial_{\mu} A_{\nu}^{\prime}-\partial_{\nu} A_{\mu}^{\prime}$ and $\tilde{F}^{\mu \nu} \equiv \frac{1}{2} \varepsilon^{\mu \nu \rho \sigma} F_{\rho \sigma}$. Remarkably, Eq. (35) is formally the same as the action of axion electrodynamics, and the screw torsion plays the role of the derivative of the vacuum angle: $S_{\mu} \sim \partial_{\mu} \theta$.

The functional derivative of the action (35) with respect to $A_{\mu}$ gives rise to the vector current,

$$
J^{\mu}=\frac{\eta_{1}}{2 \pi^{2}} S_{\nu} \tilde{F}^{\mu \nu}
$$

This equation summarizes multiple torsion-induced phenomena. The temporal component represents an anomalous charge density

$$
n=\frac{\eta_{1}}{2 \pi^{2}} \boldsymbol{S} \cdot \boldsymbol{B},
$$

resembling the Witten effect [57], in which magnetic flux traversing the gradient of the vacuum angle induces the extra charge. Thus, we call Eq. (37) the torsional Witten effect. On the other hand, the spatial component of the current reads

$$
\boldsymbol{J}=\frac{\eta_{1}}{2 \pi^{2}}\left(S_{\tau} \boldsymbol{B}+\boldsymbol{S} \times \boldsymbol{E}\right) .
$$

The first term is the torsional realization of the chiral magnetic effect [1] in which $S_{\tau}$ acts as the axial chemical potential. We thereupon designate it as the torsional magnetic effect. The second term is a current perpendicular to the electric field, which we name the torsional Hall effect after the anomalous Hall effect [58,59].

As the parity dual of the vector current (36), the axial current is derived in parallel from the anomalous action (35) as

$$
J_{5}^{\mu}=\frac{\eta_{2}}{4 \pi^{2}} E_{\nu} \tilde{F}^{\mu \nu} .
$$

Given that the torsion is mimicked by lattice dislocation [45], this relation would be useful for condensed matter experiments that create chirality imbalance without axial chemical potential.

\section{CONCLUSION}

We calculate the torsion-induced current at finite temperature, density, and curvature for the general Einstein-Cartan gravity theory. The axial current at zero temperature and density reveals the relation between the CTE and NiehYan's topological invariant. The chiral current at finite temperature and density features a rather nontrivial dependence on temperature and density, distinguished from the quadratic dependence on $T$ and $\mu_{ \pm}$in the CVE.

Our work not only has theoretical significance but also phenomenological implications. It has been proposed that torsion can be realized as lattice dislocation, indicating that the torsion-induced current is experimentally verifiable. The interaction between torsion and electromagnetic field demonstrates torsion as an alternative to the axial chemical potential for the production of chirality imbalance, heralding broader physical contexts for the study of chiral transport phenomena. The analogy between torsional electrodynamics and axion electrodynamics substantiates that novel topological effects in the latter can exist in a torsional spacetime even without a vacuum angle. One interesting example is the recently discovered 
axionic Casimir force that proves anomalously repulsive in Ref. [60].

Based on this paper, several future directions can be explored. For example, we have truncated the result to the leading order of both torsion and curvature. The generalization to higher orders would fully clarify the relation between the torsion-induced current and the axial anomaly in the Einstein-Cartan gravity theory. Also, we have treated the torsion as a background field, and the extension to dynamical torsion would be a challenging yet intriguing future task. Finally, we have restricted our discussion to noninteracting fermions. Further incorporating fermionic interactions will reveal whether there are higher loop corrections of the CTE.

\section{ACKNOWLEDGMENTS}

The authors thank Kenji Fukushima, Kazuya Mameda, and Arata Yamamoto for beneficial discussions. S. I. and Z. Q. were supported by the Grant-in-Aid for JSPS Fellows Grants No. 19J22323 and No. 20J20974.

\section{APPENDIX A: INTEGRALS IN ZERO TEMPERATURE AND DENSITY}

We supply details for the calculation of Eq. (20). Up to the leading orders of mass and curvature, Eq. (19) involves the following integrals calculated with the hard cutoff at $k^{2}=\Lambda^{2}$ :

$$
\begin{gathered}
\int^{\Lambda} \frac{d^{4} k}{(2 \pi)^{4}} \frac{1}{\left(k^{2}+m^{2}\right)^{3}}=\frac{1}{32 \pi^{2} m^{2}}, \\
\int^{\Lambda} \frac{d^{4} k}{(2 \pi)^{4}} \frac{1}{\left(k^{2}+m^{2}\right)^{2}}=\frac{1}{16 \pi^{2}}\left[\log \left(1+\frac{\Lambda^{2}}{m^{2}}\right)-1\right], \\
\int^{\Lambda} \frac{d^{4} k}{(2 \pi)^{4}} \frac{k^{2}}{\left(k^{2}+m^{2}\right)^{3}}=\frac{1}{16 \pi^{2}}\left[\log \left(1+\frac{\Lambda^{2}}{m^{2}}\right)-\frac{3}{2}\right], \\
\int^{\Lambda} \frac{d^{4} k}{(2 \pi)^{4}} \frac{k^{2}}{\left(k^{2}+m^{2}\right)^{2}} \\
=\frac{1}{16 \pi^{2}}\left[\Lambda^{2}-2 m^{2} \log \left(1+\frac{\Lambda^{2}}{m^{2}}\right)+m^{2}\right] .
\end{gathered}
$$

These formulas lead to the result (20).

\section{APPENDIX B: INTEGRALS IN FINITE TEMPERATURE AND DENSITY}

We provide a concrete derivation of Eq. (31). With the expansion (29) applied, the current (30) equals

$$
J_{ \pm}^{z}= \pm 2 \eta_{1} S^{z}\left[2 I_{2}^{z}-I_{1}+\frac{R}{6}\left(2 I_{3}^{z}-I_{2}\right)\right],
$$

where we have defined, for convenience, the following sum integrals:

$$
\begin{aligned}
I_{n} & \equiv T \sum_{\omega_{n}} \int \frac{d^{3} \boldsymbol{k}}{(2 \pi)^{3}} \frac{1}{\left(K_{ \pm}^{2}\right)^{n}}, \\
I_{n}^{z} & \equiv T \sum_{\omega_{n}} \int \frac{d^{3} \boldsymbol{k}}{(2 \pi)^{3}} \frac{k_{z}^{2}}{\left(K_{ \pm}^{2}\right)^{n}} .
\end{aligned}
$$

We adopt the dimensional regularization $d^{3} \boldsymbol{k} /(2 \pi)^{3} \rightarrow$ $M^{3-d} d^{d} \boldsymbol{k} /(2 \pi)^{d}$ with the number of dimensions $d=$ $3-2 \epsilon$ and the scale parameter $M$. Then, we carry out the momentum integrals:

$$
\begin{gathered}
I_{n}=\frac{M^{3-d} \Gamma\left(n-\frac{d}{2}\right)}{(4 \pi)^{\frac{d}{2}} \Gamma(n)} T \sum_{\omega_{n}}\left(K_{ \pm \tau}^{2}\right)^{\frac{d}{2}-n}, \\
I_{n}^{z}=\frac{1}{2(n-1)} I_{n-1} .
\end{gathered}
$$

After some algebra, the Matsubara sum amounts to

$$
\begin{aligned}
\sum_{\omega_{n}}\left(K_{ \pm \tau}^{2}\right)^{\frac{d}{2}-n}= & (2 \pi T)^{d-2 n} \\
& \times\left[\zeta\left(-d+2 n, \frac{1}{2}+i \frac{\mu_{ \pm}}{2 \pi T}\right)+\text { c.c. }\right],
\end{aligned}
$$

where $\zeta(z, a)$ denotes the Hurwitz zeta function. The integrals $I_{1}$ and $I_{2}^{z}$ have no divergence at $\epsilon=0$ and directly read

$$
\begin{gathered}
I_{1}=-\frac{\mu_{ \pm}^{2}}{8 \pi^{2}}-\frac{T^{2}}{24}+O(\epsilon), \\
I_{2}^{z}=-\frac{\mu_{ \pm}^{2}}{16 \pi^{2}}-\frac{T^{2}}{48}+O(\epsilon) .
\end{gathered}
$$

On the other hand, the integrals $I_{2}$ and $I_{3}^{z}$ diverge at $\epsilon=0$ and therefore need regularization. We exploit the Laurent series expansion of the Hurwitz zeta function:

$$
\zeta(1+2 \epsilon, z)=\frac{1}{2 \epsilon}-\psi(z)+\mathcal{O}(\epsilon) .
$$

In this way, we derive

$I_{2}=\frac{1}{16 \pi^{2}}\left[\frac{1}{\bar{\epsilon}}+2 \log \left(\frac{M}{4 \pi T}\right)-F\left(\frac{\mu_{ \pm}}{2 \pi T}\right)\right]+O(\epsilon)$,

where the definition of the function $F(z)$ has been clarified in Eq. (32) and the constant is defined as

$$
\frac{1}{\bar{\epsilon}}=\frac{1}{\epsilon}-\gamma_{\mathrm{E}}+\log (4 \pi) .
$$

Following the modified minimal subtraction scheme, we subtract the infinity as well as the logarithmic term in Eq. (B10) and obtain the final result 


$$
\begin{aligned}
& I_{2}=-\frac{1}{16 \pi^{2}} F\left(\frac{\mu_{ \pm}}{2 \pi T}\right), \\
& I_{3}^{z}=-\frac{1}{64 \pi^{2}} F\left(\frac{\mu_{ \pm}}{2 \pi T}\right) .
\end{aligned}
$$

Eventually, one can easily attain the chiral current (31) by plugging the sum integrals (B7), (B8), (B12), and (B13) into the formula (B1).

Notably, though the temperature $T$ appears in the denominator of the variable of $F(z)$, taking the zerotemperature limit $T \rightarrow 0$ does not incur a singularity because the digamma function converges for a variable with a large imaginary part. To elaborate this point, we perform the asymptotic expansion of the digamma function

$$
\psi(z)=\log z-\frac{1}{2 z}-\frac{1}{12 z^{2}}+\cdots,
$$

which leads to

$$
I_{2}=\frac{1}{16 \pi^{2}}\left(\frac{1}{\bar{\epsilon}}+2 \log \frac{\pi M}{\mu_{ \pm}}+\frac{\pi^{2} T^{2}}{3 \mu_{ \pm}^{2}}\right)+\cdots
$$

This equation illuminates the proper way to examine the low- or zero-temperature limit of our result (31). By comparison, one can analyze the small density limit via the Taylor expansion of Eq. (31) with respect to $\mu_{ \pm}$ straightforwardly.
[1] K. Fukushima, D. E. Kharzeev, and H. J. Warringa, Phys. Rev. D 78, 074033 (2008).

[2] D. T. Son and P. Surowka, Phys. Rev. Lett. 103, 191601 (2009).

[3] D. Kharzeev, J. Liao, S. Voloshin, and G. Wang, Prog. Part. Nucl. Phys. 88, 1 (2016).

[4] A. Flachi and K. Fukushima, Phys. Rev. D 98, 096011 (2018).

[5] S. L. Adler, Phys. Rev. 177, 2426 (1969); J. Bell and R. Jackiw, Nuovo Cimento A 60, 47 (1969).

[6] G. Basar and G. V. Dunne, The Chiral Magnetic Effect and Axial Anomalies, Lecture Notes Physics (Springer, Berlin, Heidelberg, 2013), Vol. 871, pp. 261-294.

[7] R. Delbourgo and A. Salam, Phys. Lett. B 40, 381 (1972); T. Eguchi and P. G. Freund, Phys. Rev. Lett. 37, 1251 (1976); L. Alvarez-Gaume and E. Witten, Nucl. Phys. B234, 269 (1984).

[8] K. Landsteiner, E. Megias, and F. Pena-Benitez, Phys. Rev. Lett. 107, 021601 (2011).

[9] G. Basar, D. E. Kharzeev, and I. Zahed, Phys. Rev. Lett. 111, 161601 (2013).

[10] D. E. Kharzeev, L. D. McLerran, and H. J. Warringa, Nucl. Phys. A803, 227 (2008).

[11] Y. Burnier, D. E. Kharzeev, J. Liao, and H.-U. Yee, Phys. Rev. Lett. 107, 052303 (2011).

[12] L. Adamczyk et al. (STAR Collaboration), Phys. Rev. Lett. 113, 052302 (2014).

[13] L. Adamczyk et al. (STAR Collaboration), Phys. Rev. Lett. 114, 252302 (2015).

[14] V. Khachatryan et al. (CMS Collaboration), Phys. Rev. Lett. 118, 122301 (2017).

[15] S. Shi, Y. Jiang, E. Lilleskov, and J. Liao, Ann. Phys. (Amsterdam) 394, 50 (2018).

[16] X.-L. Qi, T. L. Hughes, and S.-C. Zhang, Phys. Rev. B 82, 184516 (2010).

[17] S. Ryu, J. E. Moore, and A. W. Ludwig, Phys. Rev. B 85, 045104 (2012).
[18] J. Wang, B. Lian, and S.-C. Zhang, Phys. Scr. T164, 014003 (2015).

[19] A. Sekine, Phys. Rev. B 93, 094510 (2016).

[20] S. Higashikawa, M. Nakagawa, and M. Ueda, Phys. Rev. Lett. 123, 066403 (2019).

[21] C. Yue, Y. Xu, Z. Song, Y.-M. Lu, H. Weng, C. Fang, and X. Dai, Nat. Phys. 15, 577 (2019).

[22] A. Zyuzin and A. Burkov, Phys. Rev. B 86, 115133 (2012).

[23] G. Xu, H. Weng, Z. Wang, X. Dai, and Z. Fang, Phys. Rev. Lett. 107, 186806 (2011).

[24] J. Xiong, S. K. Kushwaha, T. Liang, J. W. Krizan, M. Hirschberger, W. Wang, R. J. Cava, and N. P. Ong, Science 350, 413 (2015).

[25] Q. Li, D. E. Kharzeev, C. Zhang, Y. Huang, I. Pletikosic, A. Fedorov, R. Zhong, J. Schneeloch, G. Gu, and T. Valla, Nat. Phys. 12, 550 (2016).

[26] M. Chernodub and M. Zubkov, Phys. Rev. B 95, 115410 (2017).

[27] A. Burkov, J. Phys. Condens. Matter 27, 113201 (2015).

[28] J. Charbonneau and A. Zhitnitsky, J. Cosmol. Astropart. Phys. 08 (2010) 010.

[29] M. Kaminski, C.F. Uhlemann, M. Bleicher, and J. Schaffner-Bielich, Phys. Lett. B 760, 170 (2016).

[30] G. Sigl and N. Leite, J. Cosmol. Astropart. Phys. 01 (2016) 025.

[31] D. B. Kaplan, S. Reddy, and S. Sen, Phys. Rev. D 96, 016008 (2017).

[32] M. Dvornikov, V. Semikoz, and D. Sokoloff, Phys. Rev. D 101, 083009 (2020).

[33] A. Boyarsky, J. Frohlich, and O. Ruchayskiy, Phys. Rev. Lett. 108, 031301 (2012).

[34] E. Gorbar, I. Rudenok, I. Shovkovy, and S. Vilchinskii, Phys. Rev. D 94, 103528 (2016).

[35] P. Pavlović, N. Leite, and G. Sigl, Phys. Rev. D 96, 023504 (2017).

[36] N. Yamamoto, Phys. Rev. D 93, 065017 (2016).

[37] N. Yamamoto, Phys. Rev. D 93, 125016 (2016). 
[38] N. Yamamoto and D.-L. Yang, Astrophys. J. 895, 1 (2020).

[39] Z. Khaidukov and M. Zubkov, JETP Lett. 108, 670 (2018).

[40] F. Hehl, P. Von Der Heyde, G. Kerlick, and J. Nester, Rev. Mod. Phys. 48, 393 (1976).

[41] I. Shapiro, Phys. Rep. 357, 113 (2002).

[42] H. Nieh and M. Yan, Ann. Phys. (N.Y.) 138, 237 (1982); J. Math. Phys. (N.Y.) 23, 373 (1982); H. Nieh and C. Yang, Int. J. Mod. Phys. A 22, 5237 (2007).

[43] J. Nissinen, Phys. Rev. Lett. 124, 117002 (2020); J. Nissinen and G. Volovik, arXiv:1909.08936; Pis'ma Zh. Eksp. Teor. Fiz. 110, 797 (2019) [JETP Lett. 110, 789 (2019)].

[44] Z.-M. Huang, B. Han, and M. Stone, Phys. Rev. B 101, 125201 (2020); 101, 165201 (2020); Z.-M. Huang and B. Han, arXiv:2003.04853.

[45] S. Imaki and A. Yamamoto, Phys. Rev. D 100, 054509 (2019).

[46] F. de Juan, A. Cortijo, and M. A. Vozmediano, Nucl. Phys. B828, 625 (2010).

[47] T. L. Hughes, R. G. Leigh, and E. Fradkin, Phys. Rev. Lett. 107, 075502 (2011).

[48] T. L. Hughes, R. G. Leigh, and O. Parrikar, Phys. Rev. D 88, 025040 (2013).

[49] O. Parrikar, T. L. Hughes, and R. G. Leigh, Phys. Rev. D 90, 105004 (2014).
[50] G. Volovik and M. Zubkov, Ann. Phys. (Amsterdam) 340, 352 (2014).

[51] H. Sumiyoshi and S. Fujimoto, Phys. Rev. Lett. 116, 166601 (2016).

[52] Y. You, G. Y. Cho, and T. L. Hughes, Phys. Rev. B 94, 085102 (2016).

[53] Z.-M. Huang, L. Li, J. Zhou, and H.-H. Zhang, Phys. Rev. B 99, 155152 (2019).

[54] Y. Ferreiros, Y. Kedem, E. J. Bergholtz, and J. H. Bardarson, Phys. Rev. Lett. 122, 056601 (2019).

[55] T. Bunch and L. Parker, Phys. Rev. D 20, 2499 (1979); L. E. Parker and D. Toms, Quantum Field Theory in Curved Spacetime: Quantized Field and Gravity, Cambridge Monographs on Mathematical Physics (Cambridge University Press, Cambridge, England, 2009).

[56] Y. Obukhov, Phys. Lett. B 108, 308 (1982); Nucl. Phys. B212, 237 (1983).

[57] E. Witten, Phys. Lett. 86B, 283 (1979).

[58] T. Jungwirth, Q. Niu, and A. MacDonald, Phys. Rev. Lett. 88, 207208 (2002); Z. Fang, N. Nagaosa, K. S. Takahashi, A. Asamitsu, R. Mathieu, T. Ogasawara, H. Yamada, M. Kawasaki, Y. Tokura, and K. Terakura, Science 302, 92 (2003).

[59] F. Haldane, Phys. Rev. Lett. 93, 206602 (2004).

[60] K. Fukushima, S. Imaki, and Z. Qiu, Phys. Rev. D 100, 045013 (2019). 